\title{
Therapy-related myelodysplastic syndrome after successful treatment of acute promyelocytic leukemia: case report and literature review
}

\author{
Mihaela Cîrstea ${ }^{1, *}$, Adriana Coliță ${ }^{1}$, Bogdan Ionescu ${ }^{1}$, \\ Alexandra Ghiaur ${ }^{1}$, Didona Vasilescu ${ }^{1}$, Camelia Dobrea ${ }^{1}$, \\ Cerasela Jardan ${ }^{1}$, Mihaela Dragomir ${ }^{1}$, Anca Gheorghe ${ }^{1}, Z_{\text {sofia Várady }}{ }^{\text {, }}$ \\ Anca Roxana Lupu ${ }^{2}$, Daniel Coriu ${ }^{1}$

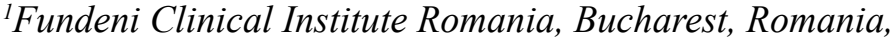 \\ ${ }^{2}$ Coltea Hospital, Bucharest, Romania
}

\begin{abstract}
In the 2016 revision of the World Health Organization classification the term therapy-related myeloid neoplasia $(t-M N)$ defines a subgroup of acute myeloid leukemia (AML) comprising patients who develop myelodysplastic syndrome (MDS-t) or acute myeloid leukemia (AML-t) after treatment with cytotoxic and/or radiation therapy for various malignancies or autoimmune disorders. We report the case of a 36 year old patient with $t-M N(t-M D S)$ after achieving complete remission (CR) of a PML-RARA positive acute promyelocytic leukemia (APL) at 32 months after diagnosis. Initially classified as low risk APL and treated according to the AIDA protocol - induction and 3 consolidation cycles - the patient achieved a complete molecular response in September 2013 and started maintenance therapy. On follow-up PML-RARA transcript remained negative. In January 2016 leukopenia and thrombocytopenia developed and a peripheral blood smear revealed hypogranular and agranular neutrophils. Immunophenotyping in the bone marrow aspirate identified undifferentiated blast cells that did not express cytoplasmic myeloperoxidase. The cytogenetic study showed normal karyotype. The molecular biology tests not identified PMLRARA transcript. A diagnosis of t-MDS (AREB-2 - WHO 2008) was established. Treatment of AML was started with 2 " $3+7$ " regimens and 1 MEC cycle. Two months from diagnosis, while in CR, an allogeneic HSCT from an unrelated HLA compatible donor was performed after myeloablative regimen. An unfavorable clinical evolution was followed by death on day 9 after transplantation. The occurrence of $t$-MNs during CR of APL represents a particular problem in terms of follow-up and differential diagnosis of relapse and constitutes a dramatic complication for a disease with a favorable prognosis.

This work was supported by the grants PN 41-087/PN2-099 from the Romanian Ministry of Research and Technology.

Keywords: acute promyelocytic leukemia, therapy-related myeloid neoplasms, allogeneic hematopoietic stem cell transplantation.
\end{abstract}

Received: 19 $9^{\text {th }}$ January 2017; Accepted: $18^{\text {th }}$ March 2017; Published: $3^{\text {th }}$ April 2017

* Corresponding author: Mihaela Cîrstea, Department of Onco-hematology, Fundeni Clinical Institute, Bucharest, Romania, e-mail: mihaelamustata84@yahoo.com 


\section{Introduction}

The term "therapy-related myeloid neoplasms" (t-MNs), proposed in the World Health Organization 2008 classification and recognized by the 2016 revision, defines a distinct category that includes patients who develop myeloid neoplasms after therapy with cytostatic agents and/or radiation therapy [1,2]. t-MNs are further subdivided into therapy-related myelodysplastic syndromes (t-MDS) and therapy-related acute myeloid leukemia (t-AML). The clinical evolution is progressive from t-MDS to t-AML and is characterized by resistance to the conventional therapy used for de novo acute leukemia [3]. The family history should be investigated especially regarding cancer susceptibility [4].

The WHO criteria $(2008,2016)$ for the diagnosis of t-MNs are based on: a) patient history of anterior exposure to cytotoxic agents or radiation, b) laboratory tests related to MDS and AML (bone marrow blasts $<20 \%=$ MDS; bone marrow blasts $\geq 20 \%=$ AML), c) cytogenetic and molecular abnormalities relevant to therapy and outcome $[1,2]$.

$\mathrm{t}-\mathrm{MNs}$ are a direct consequence of mutational events induced by therapy with cytostatic agents used for the treatment of the primary neoplasm [4]. Some individuals may have an inherited predisposition due to gene polymorphisms that affect drug metabolism or DNA repair mechanisms [3]. Mutations in proto-oncogenes have been identified in a number of t-MNs cases [5].

t-MNs have been acknowledged as clinical syndromes that arise as a late complication of cytotoxic therapy $[1,3]$. The latency period between the diagnosis of the primary neoplasm and t-MNs varies from between a couple of months to years and depends on the cumulative dose, dose-intensity and the type of preceding chemotherapy/radiation therapy [6]. There are two subcategories of t-MNs. The most frequent type of t-MN arises 5-10 year after exposure to alkylating agents and/or radiation and was initially associated with unbalanced genetic anomalies, usually involving chromosomes 5 and/or 7. A second category of t-MNs appears after a latency period of 1-5 years, after therapy with topoisomerase II inhibitors (etoposide, doxorubicin, mitoxantrone, idarubicin) and has been tied to the presence of balanced chromosomal translocations implicating 11q23 (MLL), 21q22 (RUNX1). In the WHO classification (2008, 2016) t-MNs form a single entity no longer dependent on the therapeutic agents.

t-MNs have been more often associated with treatment of solid tumors and malignant lymphomas and less with the treatment of acute leukemia $[6,7]$.

Acute promyelocytic leukemia (APL) is a subtype of AML characterized by a particular morphology of the leukemic blasts (abnormal promyelocytes), a life threatening hemorrhagic syndrome caused by hyperfibrinolysis, disseminated intravascular coagulation(DIC) and thrombocytopenia, a characteristic cytogenetic abnormality: translocation $(15 ; 17)$ that leads to the fusion of the promyelocytic leukemia gene (PML) and the retinoic acid alpha receptor gene (RARA) resulting in the PML/RARA protein (marker of the disease). APL presents a particular sensitivity to the differentiating effect of alltrans-retinoic acid (ATRA) and to the apoptotic effect of arsenic trioxide (ATO) by targeting and inducting differentiation of leukemia initiating cells (LIC) [8,9]. New data supports the view that the main mechanism of action of ATRA and ATO in eliminating leukemia initiating cells is by degrading the PML-RARA gene fusion product [10]. In the $2016 \mathrm{WHO}$ revision, in order to highlight the importance of the PML/RARA fusion - that may be cryptic and can be the result of complex cytogenetic rearrangements different 
from $\mathrm{t}(15 ; 17)(\mathrm{q} 24.1 \mathrm{q} 21.2)$ - APL with these particular abnormalities has been renamed APL with PML-RARA [2].

By introducing the combination regimen (ATRA + anthracycline-based chemotherapy) and ATRA+ATO ("chemotherapy free" regimen) as front line treatment, the prognosis of APL has changed radically. APL has been transformed from a rapidly fatal disease to a type of "curable" leukemia [8,11]. Treatment based on ATRA + anthracycline (idarubicin) -AIDA protocol - introduced through GIMEMA and PETHEMA and adapted to risk categories - is widely in use and has allowed an increase in the survival rates $[12,13,14,15]$. The analysis of the AIDA study has shown an increase in the 6-year overall survival (OS) of $87.4 \%$ and a disease free survival (DFS) of $85.6 \%$ and cumulative incidence of relapse (CIR) 10.7\% [14]. An analysis of an ATRA + ATO trial set between 2013 and 2016 has shown a 30,5-36 months OS of 93\%-99,1\%, event-free survival (EFS) 91\%$98 \%$, cumulative incidence of relapse (CIR) $1-1,1 \%[16,17,18]$. The shift to first-line therapy of ATRA + ATO (chemotherapy free) still poses ATO related problems: dose calculation, induction and consolidation regimens, the cost of the drug, long term toxicity (neurologic effects, liver damage), ease of administration (advantages of a drug that may be administered orally as opposed to intravenously).

In the last couple of years, in the specialty literature, there have been several case reports of t-MNs after successful treatment for APL with regimens including ATRA + anthracycline or ATRA + ATO [7,19,20]. t-MDS and t-AML after CR of APL have become an emerging problem [21].

$\mathrm{t}$-MNs in APL ( $\mathrm{t}-\mathrm{MDS} / \mathrm{t}-\mathrm{AML}$ ) are treatment resistant and a limiting factor in terms of favorable outcome of this particular type of leukemia. t-MNs (t-MDS/t-AML) must be distinguished from relapses which may lead to a new complete remission through the use of ATO \pm auto/allogeneic stem-cell transplantation.

\section{Case report}

A 31 year-old female nurse without a significant medical history, was admitted on April 2013 to Fundeni Clinical Institute - Center of Hematology and Bone Marrow Transplant with leukopenia and neutropenia diagnosed a couple of days prior to the admission. Her family history included the father with pulmonary neoplasia.

At diagnosis (on April 2013) she presented with relatively good general condition, afebrile, with some ecchymosis on the legs, genital bleeding, rare staphylococcal facial lesions, no palpable lymphadenopathies or organomegaly. Laboratory tests revealed: mild normochromic anemia (hemoglobin $11.3 \mathrm{~d} / \mathrm{dl}$, hematocrit $32.2 \%$, MCV 88 fl), leukopenia with severe neutropenia (WBC of $970 / \mu \mathrm{L}$ with segmented neutrophils $250 / \mu \mathrm{L}$ ), mild thrombocytopenia (platelets $73,000 / \mu \mathrm{L}$ ). Coagulation tests within normal ranges for fibrinogen $(203 \mathrm{mg} /$ dl), prothrombin activity 94\%, antithrombin III $80 \%$ and an increased value of D-Dimers $(1800 / \mu \mathrm{L})$ and positive for fibrin degradation products. Bone marrow aspirate (BMA): 19$20 \%$ myeloblasts, $28-30 \%$ promyelocytes with multiple granulations and Auer rods in "bundles". Conclusion: acute promyelocytic leukemia. No elements of dysplasia were noted (Figure 1). Bone marrow immunophenotyping identified a population of CD45 positive cells, with moderate to high internal complexity $(60 \%)$ expressing CD38, MPO, CD33, CD117, CD15士, CD123, CD34 - and HLA-DR-. Conclusion: proliferation of immature myeloid cells, suggestive for an acute promyelocytic leukemia.

FISH test: the presence of the PML/RARA fusion gene as a result of the $(15 ; 17)$ translocation (q24.1; q21.2) evident in $43 \%$ of the analyzed nuclei (Figure 2). 


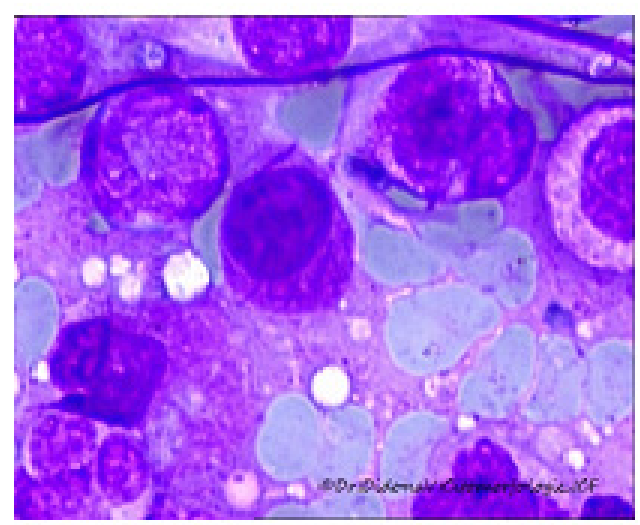

4

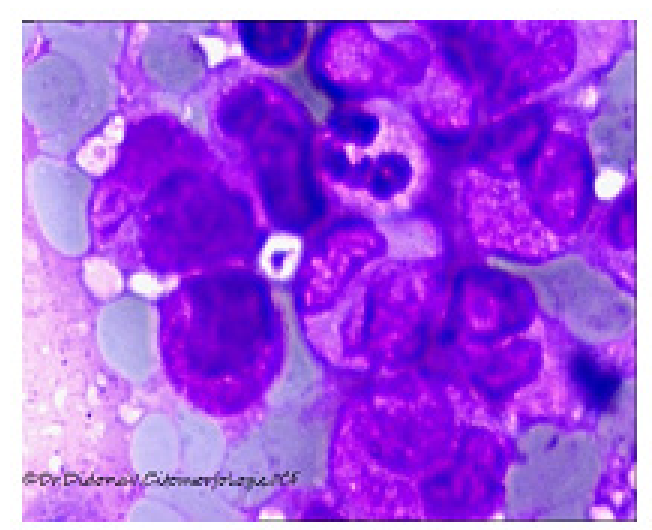

B

Figure 1. Bone marrow smear (MGG stain, ob 100x, oil immersion): A, B (april 2013) - abnormal promyelocytes

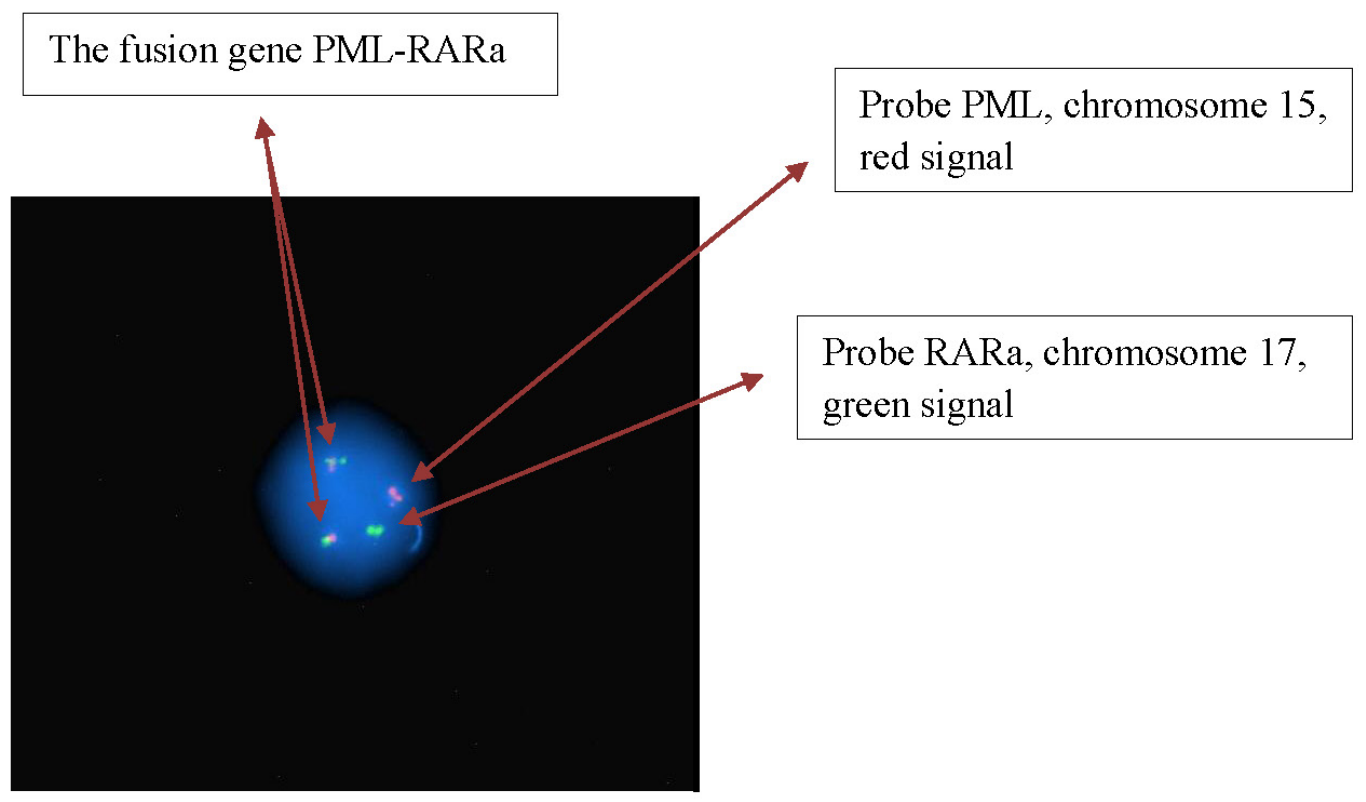

Figure 2. FISH test: nuc ish (PMLx3), (RARAx3) (PML con RARAx2) 


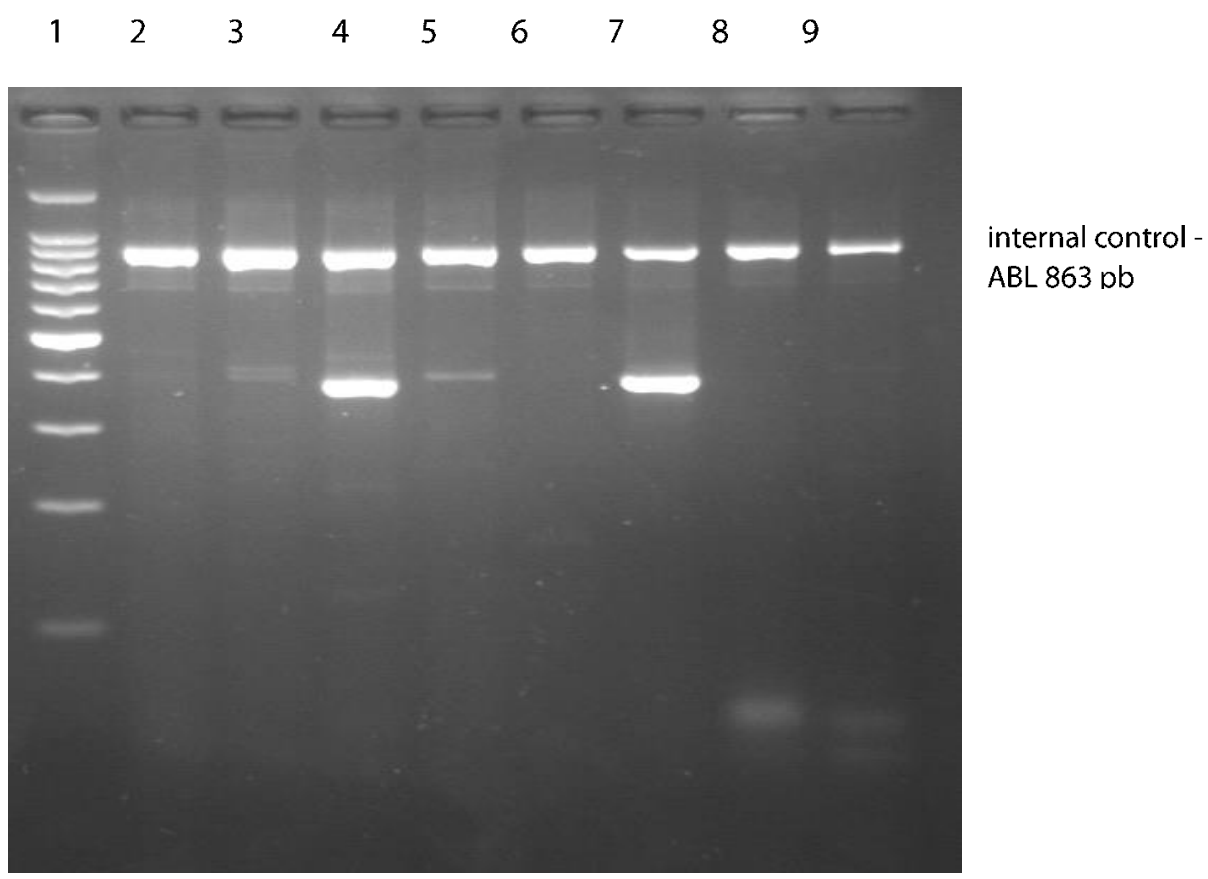

Figure 3. Agarose gel electrophoresis stained with ethidium bromide showing amplification products, representing the first step in the diagnosis of acute leukemia (technique: RNA extraction from peripheral blood followed by RT-PCR and multiplex PCR).

Line 1, DNA Ladder 100 pb-Promega; Lines 2-9, patients tested to determine the type of acute leukemia: 2,4,6,8 using mixed primers for AML and 3,5,7,9 using mixed primers for ALL; Line 4, patient positive for $A M L$; Line 7, patient positive for $A L L$.

The molecular biology tests: RT-PCR positive for the PML/RARA bcr2 isoform, negative for FLT3 ITD, CBFb - MYH11, MLL AF9, NPM1, E2A-PBX1, MLL-AF4, SIS-TAL (Figure 3,4).

Based on the morphology of the bone marrow aspirate a diagnosis of acute promyelocytic leukemia (M3 - FAB) was established and emergency treatment with oral all-trans-retinoic acid (ATRA) $45 \mathrm{mg} / \mathrm{m}^{2} /$ day was initiated on the day of admission, before FISH demonstration of the translocation $(15 ; 17)$ or PML/RARA rearrangement. The patient was included in the "low risk" relapse category (Sanz 2000 score) based on a white blood cell count of $<10,000 /$ $\mu \mathrm{L}$ and a platelet count over $40,000 / \mu \mathrm{L}$, and the AIDA protocol was decided on in terms of treatment [22].

Induction was started based on the following protocol: oral ATRA $45 \mathrm{mg} / \mathrm{m}^{2} /$ day and intravenous bolus idarubicin $12 \mathrm{mg} / \mathrm{m}^{2}$ on days 2, 4, 6, and 8 with close follow-up of clinical and biological parameters. Supportive treatment with platelet transfusion and fresh frozen plasma was required during induction in order to prevent consumption coagulopathy. The bacterial infections (cutaneous staphylococcal infection, acute tonsillitis) that occurred during post-chemotherapy cytopenia required treatment with antibiotics. Promyelocyte reversion 


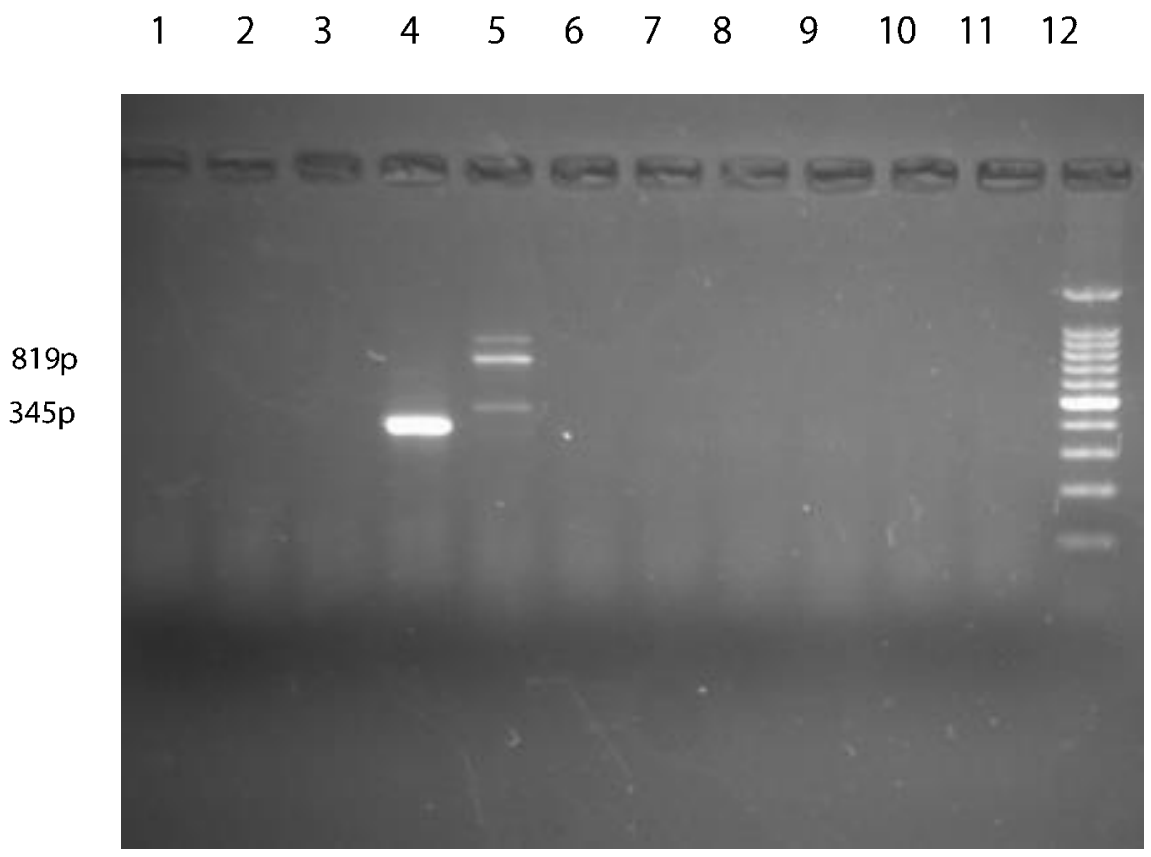

Figure 4. Agarose gel electrophoresis stainedwith ethidium bromide showing amplification products, representing the second step in the molecular diagnosis of acute leukemia. Lines 1-5: identification of fusion genes for AML; 1-AML1-ETO, 2-CBFB-MYH11 type D, 3-CBFB-MYH11 type A, 4-PML-RARa bcr1, 5-PMR-RARa bcr3; the 4th line was positive for PML-RARa bcr1 and the 5th line was positive for PML-RARa bcr3, meaning our patient was positive for PML-RARa bcr2; lines 6-11: identification of fusion genes for ALL; line 12, DNA ladder 100 pb (Promega).

was observed on the peripheral blood smear starting with the 12th day of ATRA treatment. During the third week of treatment, signs of the differentiation syndrome were evident: fever over $38^{\circ} \mathrm{C}$, weight gain and edema -symptoms that improved with dexamethasone $10 \mathrm{mg}$ iv once every 12 hours for 5 days.

Three consolidation courses according to AIDA protocol for low-risk APL: the first consolidation cycle (May - June 2013): idarubicin $5 \mathrm{mg} / \mathrm{m}^{2}$ over 4 days and ATRA 45 $\mathrm{mg} / \mathrm{m}^{2} /$ day (for 15 Days); the second cycle (July - August 2013): mitoxantrone $10 \mathrm{mg} / \mathrm{m}^{2}$ over 3 days and ATRA $45 \mathrm{mg} / \mathrm{m}^{2} /$ day (for 15 Days); third cycle (September, 2013): idarubicin $12 \mathrm{mg}$ (Day 1) and ATRA $45 \mathrm{mg} / \mathrm{m}^{2}$ (for 15 Days). After the third consolidation cycle, the patient achieved complete hematologic and molecular remission. Bone marrow aspirate showed: myeloblasts $2-3 \%$, promyelocytes $1 \%$. Nested PCR from blood and bone marrow aspirate did not identify the PML/RARA transcript. Maintenance therapy with low dose chemotherapy and ATRA consisted of oral 6-mercaptopurine $50 \mathrm{mg} / \mathrm{m}^{2} /$ day, intramuscular methotrexate $15 \mathrm{mg} / \mathrm{m}^{2} /$ weekly and oral ATRA $45 \mathrm{mg} / \mathrm{m}^{2} /$ day for 15 days every 3 months. Maintenance therapy was given between February 2014 and September 2015. Molecular biology testing every 3 months showed persistent absence of the PML/ RARA transcript. Three bone marrow aspirates showed hematologic remission without other 
abnormalities. At the follow-up in October 2015 the patient presented with good clinical condition and normal blood count $(\mathrm{Hb} 13,6 \mathrm{~g} / \mathrm{dl}, \mathrm{WBC}$ $3,400 / \mu \mathrm{L}$ : unsegmented $1 \%$, segmented $70 \%$, lymphocytes $20 \%$, monocytes $8 \%$ and platelets $236,000 / \mu \mathrm{L})$. Due to the decrease in WBC and platelet count, treatment with methotrexate and mercaptopurine was stopped in December 2015.

At follow-up on January 2016 she presented with good clinical condition, without signs of bleeding.

Laboratory tests revealed leukopenia with severe neutropenia (WBC $1,380 / \mu \mathrm{L}$, absolute neutrophil count $552 / \mu \mathrm{L}$, unsegmented $1 \%$, segmented $39 \%$, lymphocytes $42 \%$, monocytes $18 \%$ ), severe thrombocytopenia (platelets $41,000 / \mu \mathrm{L}$ ), haemoglobin within normal ranges $(\mathrm{Hb} 12 \mathrm{~g} / \mathrm{dl})$. The peripheral blood smear showed hypogranular and agranular neutrophils. Bone marrow aspirate showed slightly reduced cellularity, increased fat percentage, $\sim 16-18 \%$ myeloblasts-like agranular and granular blast cells, a decrease in the granulocytic series (45\%), hypogranular and agranular neutrophils, normal erythroid series (25\%) with normoblasts, mostly polychromatophilic and oxyphil cells, rare erythroblasts in mitosis, polymorphic megakariocytes (some with hyperlobuled nuclei and separate lobules and some small hypolobulated forms), decreased thrombocyte production. Conclusion: hypocellular bone marrow with myelodysplastic features (blast cells $<20 \%$ ) = RAEB- 2 (WHO 2008); MDS-EB 2 (WHO 2016) (Figure 5).

Bone marrow biopsy showed left shift deviation; dysplastic small hypolobulated, megacaryocytes, erythroblasts with irregular nucleus (Figure 6). The cytogenetic study was repeated in January 2016, twenty metaphase with normal karyotype (46, XX). The molecular biology tests have not identified the PML/ RARA transcript, the FLT3-ITD mutation was undetectable.
The repeated absence of a molecular anomaly (PML/RARA transcript) and $t(15 ; 17)$ excluded the possibility of an APL relapse. The neutropenia and thrombocytopenia, the presence of dysplastic changes in both the granulocytic and megakariocytic series (hypogranular and agranular neutrophils, megakariocytes some with multiple, isolated nuclei and some without lobuled nuclei), as well as $<20 \%$ blast cells in the bone marrow led to the diagnosis of myelodysplastic syndrome (MDS) - refractory anemia with excess blasts 2 (MDS-EB2) (WHO 2016 classification). The bone marrow aspirate immunophenotyping established the phenotypic profile of an undifferentiated blast cell proliferation that does not express intracytoplasmatic myeloperoxidase (icMPO), but expresses myeloid surface markers in the context of myelodysplasia.

The diagnosis of therapy-related myelodysplastic syndrome was established (t-MDS -> RAEB-2) in a patient with complete molecular remission of APL after treatment with ATRA and anthracycline-based chemotherapy. The time interval from complete remission of APL to the diagnosis of t-MDS (RAEB-2) was 28 months. When the diagnosis of t-MN was established, the patient was on month 23 of maintenance therapy (ATRA + MTX + 6-MP). Calculating the risk category for t-MDS (RAEB2), survival rate and risk of transformation to AML, the prognostic scores yielded the following results: a) International Prognostic Scoring System (IPSS): intermediate-2 risk group with a median survival of 1.2 years and a $25 \%$ risk of transformation to AML in 1.1 years; b)WHO-classification based Prognostic Scoring System (WPSS score): 3 (high risk) with a median survival of 2.2 years and a risk of transformation to AML of 0.54.

This is the case of a young patient (36 years old), with a good performance status (KPS 90$100 \%$, without comorbidities, with a diagnosis 


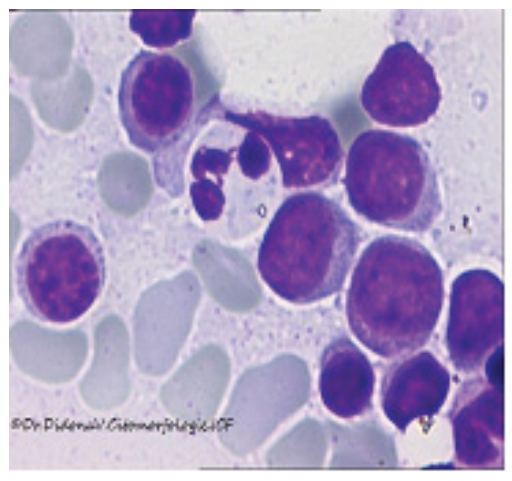

A

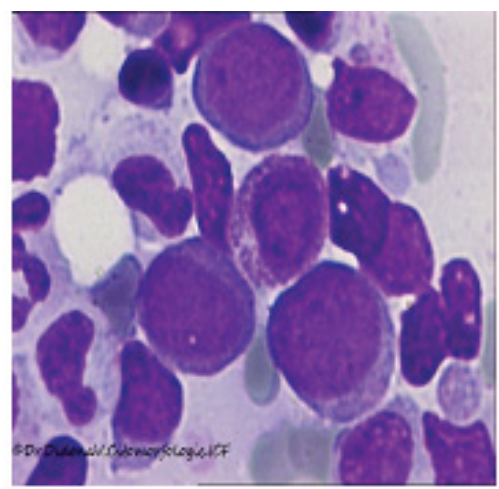

B

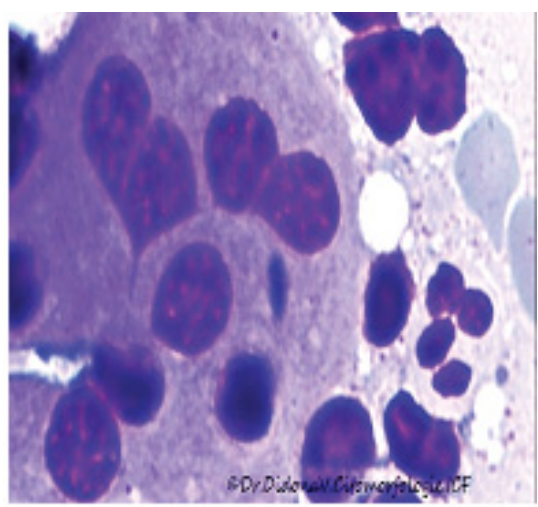

$\mathrm{C}$

Figure 5. Bone marrow smear (MGG stain, ob 100x, oil immersion) - january 2016: A - myeloblasts, agranular neutrophil; B- myeloblasts, agranular neutrophils, C- hyperlobated megakaryocytes with separate nuclei.
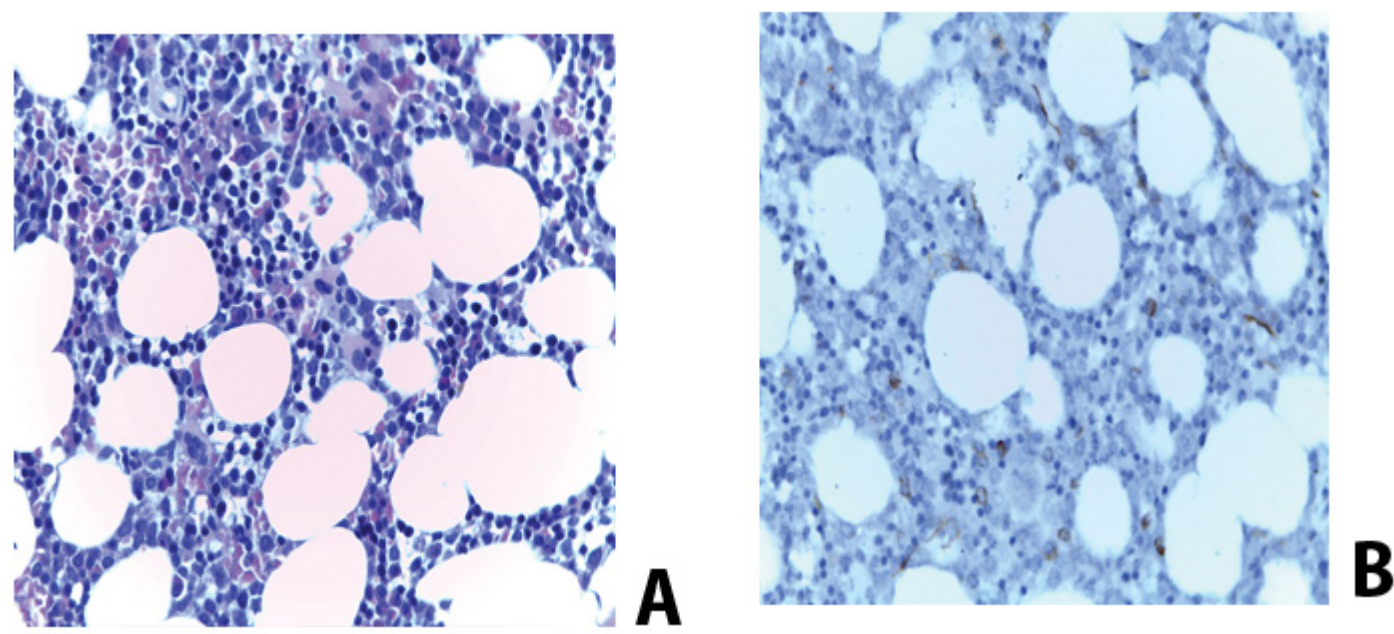

Figure 6. A - Bone marrow trephine biopsy; left shift deviation; dysplastic small hypolobulated megacaryocytes; erythroblasts with irregular nucleus. (H\&E stain, ob 40x)

B - Bone marrow trephine biopsy: isolated CD 34 positive cells; (IHC stain for CD 34, ob 40X).

of t-MDS (RAEB-2) with intermediate/high risk prognostic scores and high risk of transformation to AML. It was decided to start the AML chemotherapy in order to induce complete remission followed by allogeneic stem cell transplantation (allo-HSCT). During January 2016 - April 2016, the patient received: 2 " $3+7$ ” cycles with idarubicin $12 \mathrm{mg} / \mathrm{m}^{2}$ for three days and cytarabine $200 \mathrm{mg} / \mathrm{m}^{2} / \mathrm{zi}$ for 7 days and 1 MEC cycle (mitoxantrone $12 \mathrm{mg} / \mathrm{m}^{2} /$ day - Days 
1-5, etoposide $100 \mathrm{mg} / \mathrm{m}^{2} /$ day - Days $1-5$ and cytarabine $1000 \mathrm{mg} / \mathrm{m}^{2} /$ day - Days 1-5). The chemotherapy cycles were followed by cytopenia that required long periods of hospitalization, antibiotics, antifungal treatment and supportive care with red blood cell and platelets transfusions. At the evaluation on May 2016 the patient presented with good clinical condition, afebrile, normal leucocytes with normal neutrophils (WBC $4,160 / \mu \mathrm{L}$, unsegmented $2 \%$, segmented neutrophils $72 \%$, eosinophils $1 \%$, monocytes $12 \%)$, normal platelets $(132,000 / \mu \mathrm{L})$ and mild anemia (Hb 10.4 g/dl, Ht 35\%).

Bone marrow aspirate showed slightly decreased cellularity, 3-4\% blast cells (myeloblasts, monocytoid blasts), decrease of the granulocytic series, with maturation: hypogranular and agranular neutrophils, increase of the erythroid series (39\%), megakaryocytes present. On June 2016, the patient is admitted to the Departament of Bone Marrow Transplantation Fundeni Clinical Institute. The patient's data on evaluation before allo-HSCT: 36 year old, female patient, Karnofsky Performance Status $100 \%$, with complete remission after AML chemotherapy with 3-4\% blast cells in the bone marrow aspirate. The cytogenetic study revealed normal karyotype. The time from t-MDS diagnosis to transplant was 6 months. CMV status was negative. The donor's data mentioned 10/10 HLA matched unrelated donor, male, 30 years old, 0 negative group (patient: B positive), CMV negative. Data related to the transplant procedure: myeloablative conditioning regimen $(\mathrm{Bu} / \mathrm{Cy})$; GVHD prophylaxis with cyclosporine, MTX + ATG started on June 2016. Posttransplant status with severe mucositis, E. coli sepsis due to persistent agranulocytosis, severe thrombocytopenia, severe anemia with transfusion incidents. Despite supportive care (broad spectrum antibiotics, red blood cell and platelets transfusions, electrolyte imbalance correction) the patient's status declined progressively and death occurred on day 9 after transplantation (Table 1).

\section{Discussions}

During the last couple of years multiple reports on treatment-related neoplasia have been published: t-MDS and t-AML in patients with complete remission after APL treated by standard protocol with ATRA and anthracyclinebased chemotherapy (AIDA) [7,19,20,21,23,24].

The incidence of t-MN in APL reported in studies varies: $0.97 \%$ (6 of 617 patients) [24], $6.5 \%$ (3 of 46 patients) [21], 10.2\% (11 of 108 patients) [19]. Montesinos et al. (2010) reported $17 \mathrm{t}-\mathrm{MNs}$ diagnosed in a number of 918 patients with APL with complete remission. Of the 17 t-MNs cases: 10 were t-MDS (bone marrow blast cells $<20 \%$ ) and 7 were AML (bone marrow blast cells $>20 \%$ ). The cumulative incidence in a time frame of 6 years: 2.2\%. 6-year incidence based on risk group: low, intermediary and high were of $5.2 \%, 2.1 \%$ and $0 \%$. Another 14 patients developed a series of solid tumors (colorectal carcinoma $(n=4)$; carcinoma of the lung $(n=2)$, breast $(n=2)$, prostate $(n=1)$, kidney $(n=1)$, thyroid $(n=1)$, pancreas $(n=1)$, cervix $(n=1)$, non-Hodgkin lymphoma $(\mathrm{n}=1)$.

The higher incidence of APL in younger patients (starting with the second decade of life) is well known, and decreases after the age of 60 $[25,26,27]$. For patients who develop t-MNs during the CR of APL, the median age at APL diagnosis was 50 years old (18-68 years) [7] and 52 years (18-86 years) [19]. In the PETHEMA study, there was a higher prevalence among female patients $(11 \mathrm{~F} / 6 \mathrm{M})$ and a higher male prevalence in the Japanese study (3F/9M) [7,19].

The time interval between APL diagnosis and the development of a t-MN was 43-46 months in the PETHEMA study. Three of the 17 cases of t-MNs emerged during maintenance therapy [7]. 
Table 1. The patient's main characteristics and clinical evolution

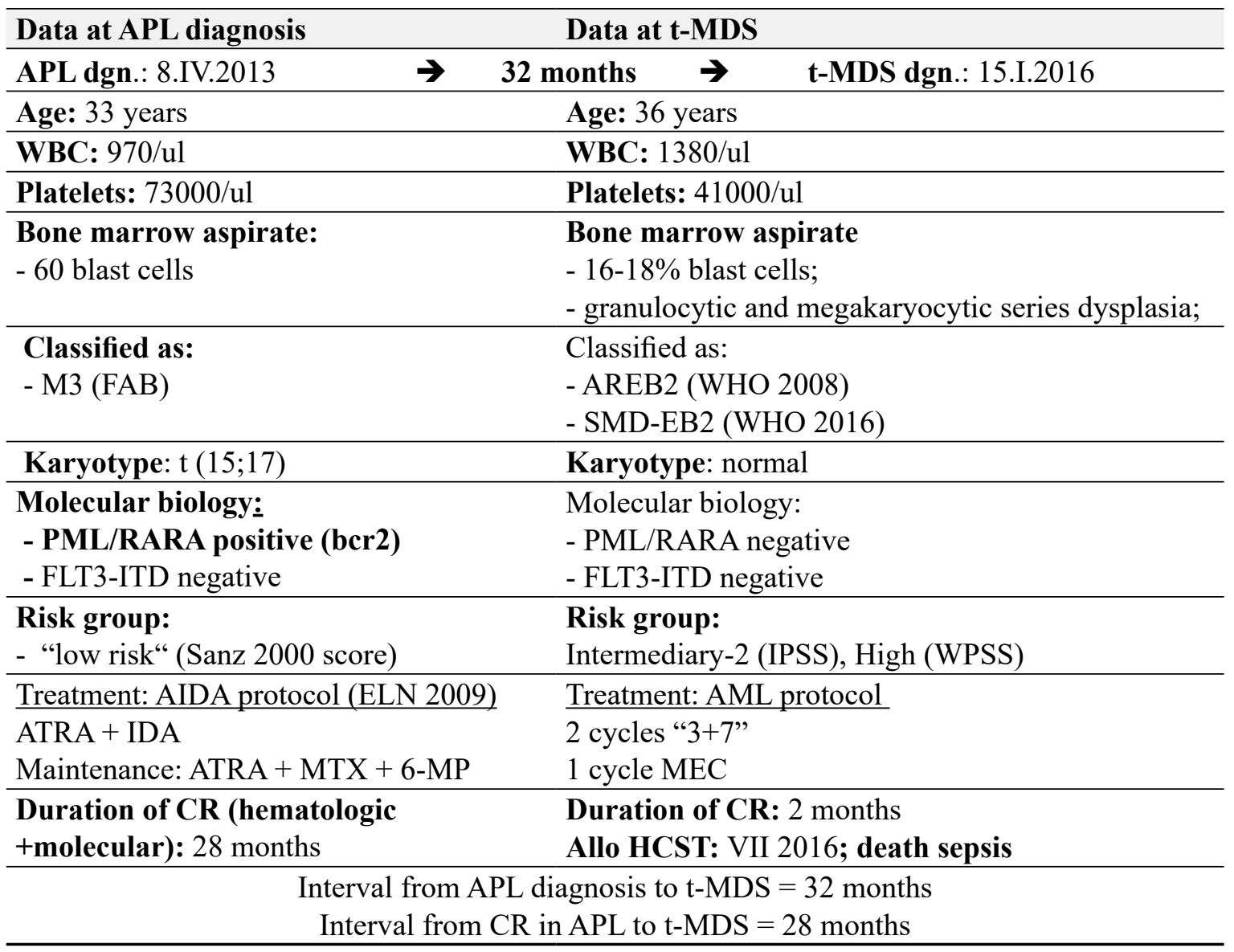

Cases of t-MNs have been reported in the second and third CR of APL [21].

A Japanese study observed the clinical and hematopoietic differences between t-MNs in CR of APL and relapses [19]. In a lot of 108 patients with APL in their first complete remission there were $11(10.2 \%)$ who developed t-MNs (t-MDS and t-AML) and 10 (9.3\%) who relapsed. The median follow-up period: 8.6 years (1.7-16.3 years). The median time from the diagnosis of ALP to t-MDS (RCMD) was 2.3 years (1.63 years) and from the diagnosis of APL to t-MDS/t-AML: 3.3 years (1.0-9.7 years) and for APL relapse: 2.6 years (0.6-10.1 years). By comparing the hematologic data from $11 \mathrm{t}-\mathrm{MN}$ cases and 10 relapse cases, the authors noted a statistically significant difference in terms of the white blood cell counts at APL diagnosis. t-MNs patients had a lower WBC (leucocytes $\leq$ $4.500 / \mu \mathrm{L})$ as opposed to those with relapse (leucocytes $>4.500 / \mu \mathrm{L})(\mathrm{P}=0,048)$. The t-MNs cases were PML/RARA-, CD34+, HLA-DR+ and the RUNX1/AML1 mutation was reported in 4 cases. Overall survival measured from the initiation of chemotherapy was significantly lower in patients with t-MNs than in those with relapse $(\mathrm{P}=0.022)$

In t-MNs after APL frequent karyotype abnormalities with poor outcomes have been identified: partial or complete deletions of 
chromosomes 5 and 7, translocations involving the MLL gene (mixed lineage leukemia) found in the 11q23 region, monosomal karyotype.

An analysis of the cytogenetic alterations in 14 of the 17 patients in the PETHEMA study has shown: partial or complete deletions of the chromosomes 5 and 7 in 9 patients; 11q23/ MLL rearrangements in 3 patients; complex karyotype ( $\geq 3$ abnormalities) in 6 patients; normal karyotype in 1 patient; monosomal karyotype in 6 patients. Translocations involving MLL/11q23, that typically correlates with topoisomerase II inhibitor therapy was encountered less frequently ( 3 cases) than $-5 /$ del $(5 q)$ and $-7 /$ del (7q) (9 cases). The partial or complete deletion of chromosomes 5 and 7 was associated in some studies with prior exposure to alkylating agents - not applicable to the studied APL patients. The ELN group [24] described 6 patients with t-MDS in CR of APL with abnormalities in chromosomes 5 and 7, despite not having received treatment with alkylating agents or radiotherapy. The observations made by researchers of the PETHEMA and the ELN group support the changes recently added by the WHO $(2008,2016)$ claiming that t-MNs can no longer be divided into 2 subcategories: alkylating agents related and topoisomerase II related. Today t-MNs are classified as a single entity.

In analyzing the $11 \mathrm{t}-\mathrm{MDS} / \mathrm{t}-\mathrm{AML}$ the Japanese group (Imagawa et al.) found: complete deletions of $7 /-7$, translocations implicating 21q 22 (RUNX1) and 11q 23 (MLL). PMLRARA negative t-MNs develop from a "receptive" hematopoietic stem cell or a normal hematopoietic progenitor cell through the accumulation of genic abnormalities induced by chemotherapy, including the RUNX1 mutation [19]. In terms of molecular biology, Imagawa et al. found mutations involving RUNX1 (4 cases) and CEPBA ( 1 case) - mutations that were not present at APL diagnosis. 3 out of 4 RUNX1 mutations were correlated with monosomy 7 . One case with a RUNX1 mutation also acquired NRAS.

The majority of $t-M N$ cases ( $t-M D S / t-A M L)$ were either low or intermediate risk APL. At APL diagnosis, the risk score for relapse (Sanz score) allows for a better choice regarding treatment, based on WBC and platelet counts. Patients at low risk receive a lower total dose of anthracyclines (idarubicin, mitoxantron) that those with high risk [15,22]. The data from literature reveals a higher risk of developing t-MNs in patients with a lower risk of relapse. Montesinos \& al. [7] mention that out of 17 patients with t-MNs, 8 were low risk (136 total patients) and 9 were intermediary risk (from a total of 280 patients). Imagawa $\&$ al. [19] remark that 9 out of 11 patients with t-MNs were low risk and 2 were high risk. These papers do not support the potential correlation between dose-intensity of topoisomerase II inhibitors and a higher prevalence of t-MNs in low risk patients who are generally treated with lower dose anthracycline regimens. The transition toward chemotherapyfree first-line treatment based on the association of ATRA + ATO in newly diagnosed low and intermediary risk cases comes as a solution in trying to lower the incidence of t-MNs [16,18].

Maintenance therapy with methotrexate and mercaptopurine may have a potential role in the development of t-MNs - as proven in children with acute lymphoblastic leukemia [28]. It is known that maintenance therapy reduces the risk of relapse. However, it increases incidence of $\mathrm{t}-\mathrm{MNs}$ in APL. By using etoposide (VP-16) in the maintenance regimen, the incidence of t-MNs increased to $10.2 \%$ in the Japanese study [19]. PCR monitoring of the PML/RARA transcript in CR of APL with low risk may be a substitute for maintenance therapy. Prevention of t-MNs is very important. t-MNs in APL represents a 
limiting factor in the outcome of a now curable form of leukemia.

t-MNs that develop over CR of APL have a poor prognosis. Cytogenetic abnormalities associated with poor outcomes (monosomal karyotype, partial or complete chromosome 5 or 7 deletions, complete karyotype) found in the majority of cases predict aggressive clinical evolution [7].

In terms of risk factors, through multivariate analysis, the PETHEMA group has highlighted the following characteristics associated with t-MN development: older age (35 years as the most significant cutoff) $(\mathrm{p}=0.008)$; low risk of relapse $(\mathrm{p}=0.008)$; high platelet count (with 40 $\mathrm{x} 10^{\wedge} 9 / 1$ as the most significant cutoff) $(\mathrm{p}=0.03)$. The multivariate analysis identified independent prognosis factors for t-MNs: age (hazard ratio $=8.89 ; \mathrm{p}=0.001)$ and relapse score(hazard ratio $=0.34 ; p=0.005$ ) [7].

The survival of t-MN patients is poor even in cases with timely diagnosis and treatment. t-MN patients are excluded for MDS/AML front line treatment trials. The PETHEMA analysis [7] involving the 17 cases of t-MNs (diagnosed among 918 APL patients in CR) may give information of prognosis and treatment. Eight of the 10 cases of t-MDS progressed to acute leukemia in 9 months after diagnosis (4-19 months) and 9 out of 10 patients deceased after a median period of 10 months (5-32 months). One patient with t-MDS (AREB1) developed acute "B" lymphoblastic leukemia (CD45-, TDT+, CD79+, CD34-, CD19+, CD10+, no myeloid markers), was treated and died after 32 month with treatment-resistant disease. Four patients with t-MDS who developed AML and a patient with t-MDS (RAEB-1) received intensive chemotherapy followed by allogeneic hematopoietic stem-cell transplantation (alloHSCT), with only one of the patients still alive at 30 months. Out of the 7 t-AML cases, one patient received only supportive care while 6 others received intensive chemotherapy. Three of the patients who achieved CR underwent alloHSCT but only one survived at 39 months. Five patients died after a median time of 10 months from the second diagnosis (2-12 months). Overall survival (OS) 2 years after the diagnosis of t-MN was $19 \%$ with a median OS of 10 months [7]. These patients' low hematopoietic stem cell reserves make standard AML therapy difficult. Drug resistance of the new malignant clone is another cause of treatment failure. Often, even cytogenetic and molecular remissions are shortened. Allogeneic stem-cell transplantation is most likely the only potentially curative treatment [29]. After analyzing the results of the EBMT group between 1981-2006, Kroger determined that only a third of the 461 patients with t-MNs, were "cured" after receiving alloHSCT. In the multivariate analysis younger patients $(<40$ years old) with normal karyotypes who were transplanted during the first CR had the most favorable prognosis. Some case series describe $20-30 \%$ survival at 3 years after alloHSCT in t-MNs.

Monitoring the $\mathrm{CR}$ in a patient with APL is done by detecting the PML/RARA transcript in the bone marrow or peripheral blood samples. The reappearance of the PML/RARA transcript is typical of molecular relapse and precedes hematologic relapse. The PETHEMA group suggested that patients treated during molecular relapse have more favorable outcomes than those treated after hematologic relapse. The timely detection of the PML/RARA transcripts and ATO treatment initiation may lead to a new CR [30,31].

Patients who develop t-MNs during the CR of APL are PML/RARA negative. Cytogenetic analysis show no evidence of $t(15 ; 17)$. Some cytogenetic aberrations may be present (complete or partial deletions of the chromosomes 5 and 
7, complex karyotype, translocations involving 11q23 (MLL) or 21q23 (RUNX1), blast cells with a different phenotype that promyelocytic blast cells at the diagnosis of APL.

During the follow-up of a patient in remission at low/intermediate risk of relapse, a closer look must be taken at the changes of the blood count (cytopenia, peripheral blood anomalies: macrocytosis, hypogranular or agranular neutrophils). In such cases obtaining a bone marrow sample is in order; karyotyping may find signs of myeloid neoplasia (MDS, AML).

\section{Conclusion}

NM-t in RC of APL are a distinct problem in terms of follow-up and differential diagnosis with disease relapse. Good survival rates occur in patients who achieve a new CR and are eligible for allo-HSCT. The possibility of developing a treatment-related myeloid neoplasm accentuates the importance of long term surveillance of APL survivors.

\section{Conflicts of interest}

The authors declare that they have no conflict of interest.
Abbrevations:
APL = acute promyelocytic leukemia
Allo-HSCT = allogeneic hematopoietic stem cell transplantation
AML = acute myeloid leukemia
ATG = anti-thymocyte globulin
ATRA $=$ all-trans retinoic acid
ATO = arsenic trioxide
$\mathrm{BM}=$ bone marrow
$\mathrm{BMB}=$ bone marrow biopsy
$\mathrm{BU} / \mathrm{CY}=$ busulfan/ cyclophosphamide
CIR = cumulative incidence of relapse

\begin{tabular}{|c|c|}
\hline CMV & $=$ cytomegalovirus \\
\hline $\mathrm{CR}$ & $=$ complete remission \\
\hline CSA & $=$ cyclosporine \\
\hline $\mathrm{DIC}$ & $\begin{aligned}= & \text { disseminated intravascular } \\
& \text { coagulation }\end{aligned}$ \\
\hline DFS & $=$ disease free survival \\
\hline EFS & $=$ event-free survival \\
\hline FAB & $=$ French-American-British \\
\hline FISH & $\begin{aligned}= & \text { fluorescent in situ } \\
& \text { hybridization }\end{aligned}$ \\
\hline GvHD & $=$ graft versus host disease \\
\hline $\mathrm{Hb}$ & $=$ haemoglobin \\
\hline $\mathrm{HE}$ & $=$ hematoxylin and eosin \\
\hline $\mathrm{Ht}$ & $=$ haematocrit \\
\hline HLA & $=$ human leucocyte antigen \\
\hline IHC & $=$ immunohistochemistry \\
\hline IPSS & $\begin{aligned}= & \text { International Prognostic } \\
& \text { Scoring System }\end{aligned}$ \\
\hline KPS & $=$ Karnofsky performance status \\
\hline LIC & $=$ leukemia- initiating cells \\
\hline MAC & $=$ myeloablative conditioning \\
\hline MDS & $=$ myelodysplastic syndrome \\
\hline MDS-EB-2 & $=$ MDS with excess blasts- 2 \\
\hline MPO & $=$ myeloperoxidase \\
\hline MTX & $=$ methothrexate \\
\hline 6-MP & $=$ mercaptopurine \\
\hline OS & $=$ overall survival \\
\hline PB & $=$ peripheral blood \\
\hline PETHEMA & $\begin{aligned}= & \text { Programa Español para el } \\
& \text { Tratamiento de Enfermedades } \\
& \text { Hematológicas }\end{aligned}$ \\
\hline PML & $=$ promyelocytic leukemia \\
\hline RAEB-2 & $\begin{aligned}= & \text { refractory anemia with excess } \\
& \text { blasts }\end{aligned}$ \\
\hline RARA & $=$ retinoic acid receptor alpha \\
\hline RCMD & $\begin{aligned}= & \text { refractory cytopenia with } \\
& \text { multilineage dysplasia }\end{aligned}$ \\
\hline t-AML & $\begin{aligned}= & \text { therapy- related acute myeloid } \\
& \text { leukemia }\end{aligned}$ \\
\hline
\end{tabular}




$\begin{array}{rlrl}\mathrm{t}-\mathrm{MDS} & = & \text { therapy- related } \\ & \text { myelodysplastic syndromes } \\ \mathrm{t}-\mathrm{MNs} & = & \text { therapy- related myeloid } \\ & \text { neoplasms } \\ \mathrm{WBC} & = & \text { white blood cell } \\ \mathrm{WHO} & = & \text { World Health Organization } \\ \mathrm{WPSS} & = & \text { WHO prognostic scoring system }\end{array}$

\section{References}

1. Vardiman JW, Arber DA, Brunning RD, Larson RA, Matutes E, Baumann I, et al. Therapy - related myeloid neoplasms in: Swerdlow SH, Campo E, Harris NL, Jaffe ES, Pileri SA, Stein H, Thiele J, Vardiman JW, editors. WHO classification of Tumours of Haematopoietic and Lymphoid Tissues. Fourth Ed. Lyon France: IARC Press 2008: 127-129.

2. Arber DA, Orazi A, Hasserjian R, Thiele J, Borowitz MJ, Michelle M, et al. The 2016 revision to the World Health Organization classification of myeloid neoplasms and acute leukemia. Blood 2016; 127:23912405. DOI: 10.1182/blood-2016-03-643544

3. Larson RA. Therapy - related myeloid neoplasms. Haematologica 2009 April;94(4):454-9. DOI: 10.3324/ haematol.2008.005157

4. Heuser M. Therapy-related myeloid neoplasms: does knowing the origin help to guide treatment? Hematology Am Soc Hematol Educ Program. 2016 Dec;(1):24-32. DOI: 10.1182/asheducation-2016.1.24

5. Churpek JE, Marquez R, Neistadt B, Claussen K, Lee MK, Churpek MM, et al. Inherited mutations in cancer succeptibility genes are common among survivors of breast cancer who develop therapy - related leukemia. Cancer. 2016 Jan;122(2):304-11. DOI: 10.1002/ cncr.29615

6. Kayser S, Dohner K, Krauter J, Köhne CH, Horst HA, Held G, et al. The impact of therapy - related acute myeloid leukemia (AML) on outcome in 2853 adult patients with newly diagnosed AML. Blood $2011 \mathrm{Feb}$; 117(7):2137-45. DOI: 10.1182/blood-2010-08-301713

7. Montesinos P, Gonzalez JD, Gonzalez J, Rayo'n C, de Lisa E, Amigo ML, et al. Therapy - Related Myeloid Neoplasms in patients with acute promyelocytic leukemia treated with all- trans retinoic acid and anthracycline based chemotherapy. J Clin Oncol. 2010 Aug;28(24):3872-9. DOI: 10.1200/JCO.2010.29.2268
8. Lo-Coco F, Cicconi L, Breccia M. Current standard treatment of acute promyelocytic leukemia. British Journal of Haematology. 2016 Mar;172(6):841-4. DOI: 10.1111/bjh. 13890

9. Zeidan AM, Gore SD. New strategies in acute promyelocytic leukemia: Moving to an Entirely Oral, Chemotherapy-Free Upfront Management Approach. Clinical Cancer Research. 2014 Oct; 20(19):4985-93. DOI: $10.1158 / 1078-0432 . C C R-13-2725$

10. Tomita A, Kiyoi H, NaoeT. Mechanisms of action and resistance to all-trans retinoic acid (ATRA) and arsenic trioxide (As2O 3) in acute promyelocytic leukemia. Int J Hematol. 2013 Jun;97(6):717-25. DOI: 10.1007/ s12185-013-1354-4

11. Wang ZY, Chen Z. Acute promyelocytic leukemia: from highly fatal to highly curable. Blood. 2008 March; 111(5):2505-14. DOI: 10.1182/blood-2007-07-102798

12. Sanz MA, Lo Coco F, Martin G, Avvisati G, Rayón C, Barbui $\mathrm{T}$, et al. Definition of relapse risk and role of nonanthracycline drugs for consolidation in patients with acute promyelocytic leukemia: a joint study of the PETHEMA and GIMEMA cooperative groups. Blood. 2000 Aug; 96:1247-53.

13. Sanz MA, Grimwade D, Tallman MS, Lowenberg B, Fenaux P, Estey EH, et al. Management of acute promyelocytic leukemia: recommandations from an expert panel on behalf of the European Leukemia Net. Blood 2009 Feb;113(9):1875-91. DOI: 10.1182/ blood-2008-04-150250

14. Sanz MA, Lo-Coco F. Modern Approaches to Treating Acute Promyelocytic Leukemia. J Clin Oncol. 2011 Feb;29(5):495-503. DOI: 10.1200/JCO.2010.32.1067

15. Lo-Coco F, Avvisati G, Vignetti M, Breccia M, Gallo E, Rambaldi A, et al. Front-line treatment of acute promyelocytic leukemia with AIDA induction followed by risk-adapted consolidation for adults younger than 61 years: results of the AIDA-2000 trial of the GIMEMA Group. Blood 2010 Oct; 116(17):3171-9. DOI: 10.1182/ blood-2010-03-276196

16. Lo-Coco F, Avvisati G, Vignetti M, Thiede C, Orlando SM, Iacobelli S, et al. Retinoic Acid and Arsenic Trioxide for Acute Promyelocytic Leukemia. N Engl J Med 2013 Jul;369(2):111-21. DOI: 10.1056/ NEJMoa1300874 
17. Platzbecker U, Avvisati G, Cicconi L, Thiede C, Paoloni F, Vignetti M, et al. Improved Outcomes With Retinoic Acid and Arsenic Trioxide Compared With Retinoic Acid and Chemotherapy in Non-High-Risk Acute Promyelocytic Leukemia: Final Results of the Randomized Italian-German APL0406 Trial. J Clin Oncol 2016 Jul;1-14.

18. Burnett AK, Russell NH, Hills RK, Bowen D, Kell J, Knapper S, et al. Arsenic trioxide and all-trans retinoic acid treatment for acute promyelocytic leukaemia in all risk groups (AML17): results of a randomised, controlled, phase 3 trial. The Lancet Oncology 2015 Oct;16(13): 1295-305. DOI: 10.1016/S14702045(15)00193-X

19. Imagawa J, Harada $Y$, Shimomura $T$, Tanaka $H$, Okikawa $\mathrm{Y}$, Hyodo $\mathrm{H}$, et al. Clinical and genetic features of therapy-related myeloid neoplasms after chemotherapy for acute promyelocytic leukemia. Blood 2010 Dec; 116(26):6018-22. DOI: 10.1182/ blood-2010-06-289389

20. Eghtedar A, Rodriquez I, Kantarjian H, O'Brien S, Daver N, Manero GG, et al. Incidence of secondary neoplasms in patients with acute promyelocytic leukemia treated with all-trans retinoic acid plus chemotherapy or with all-trans retinoic acid pluss arsenic trioxide. Leuk. Lymphoma 2015 May;56(5):1342-5. DOI: 10.3109/10428194.2014.953143

21. Latagliata R, Petti MC, Fenu S, Mancini M, Spiriti MAE, Breccia $M$, et al. Therapy-related myelodysplastic syndrome- acute myelogenous leukemia in patients treated for acute promyelocytic leukemia: an emerging problem. Blood 2002 Feb; 99(3):822-4. DOI: $10.1182 /$ blood.V99.3.822

22. Sanz MA, Montesinos P, Rayón C, Holowiecka A, de la Serna J, Milone G, et al. Risk-adapted treatment of acute promyelocytic leukemia based on alltrans retinoic acid and anthracycline with addition of cytarabine in consolidation therapy for high-risk patients: further improvements in treatment outcome. Blood.2010 Jun;115(25):5137-46. DOI: 10.1182/ blood-2010-01-266007

23. Panizo C, Patino A, Lecumberri R, Calasanz MJ, Odero $\mathrm{MD}$, Bendandi $\mathrm{M}$, et al. Secondary myelodysplastic syndrome after treatment for promyelocytic leukemia: clinical and genetic features of two cases. Cancer Genetics and Cytogenetics 2003 Jul; 143(2):178-81. DOI: $10.1016 / \mathrm{S} 0165-4608(02) 00859-2$
24. Lobe I, Rigal-Huguet F, Vekhoff A, Desablens B, Bordessoule D, Mounier C, et al. Myelodysplastic syndrome after acute promyelocytic leukemia: the European APL group experience. Leukemia. 2003 Aug;17(8):1600-4. DOI: 10.1038/sj.leu.2403034

25. Coliţă A, Nicoară S, Butoianu E, Munteanu N, Ursea C, Poppa C, et al. Acute promyelocytic leukemia. A study of 119 cases and a review of the literature. Rev Med Interna Neurol Psihiatr Neurochir Dermatovenerol Med Interna. 1988 Jan-Feb;40(1):21-32.

26. Colita A, Vasilache D, Gheorghe A, Jardan C, Jardan D, Dragomir M, et al. Acute promyelocytic leukemia: the importance of the laboratory examination for the diagnosis and the dynamic following of the answer to the treatment. Romanian Review of the Laboratory Medicine 2013 Jun; 21(2/4):S60.

27. Coombs CC, Tavakkoli M, Tallman MS: Acute promyelocytic leukemia: where did we start, where are we now, and the future. Blood Cancer Journal 2015 Feb;5, e304. DOI: $10.1038 /$ bcj.2015.25

28. Schmiegelow K, Al - Modhwahii, Andersen MK, Behrendtz M, Forestier E, Hasle H, et al. Methotrexate (6- mercaptopurine maintenance therapy influences the risk of a second malignant neoplasm after childhood acute lymphoblastic leukemia. Results from the NOPHO ALL- 92 study. Blood 2009;113:6077-884. DOI: 10.1182/blood-2008-11-187880

29. DöhnerH, Estey E, Grimwade D, Amadori S, Appelbaum FR, Büchner T, et al. Diagnosis and management of AML in adults: 2017 ELN recommendations from an international expert panel. Blood 2017 Jan;129(4):42447. DOI: 10.1182/blood-2016-08-733196

30. Watts JM, Tallman MS. Acute promyelocytic leukemia: What is the new standard of care? Blood Rev 2014 Sep; 28(5):205-12. DOI: 10.1016/j.blre.2014.07.001

31. Esteve J, Escoda L, Martín G, Rubio V, DíazMediavilla J, González M, et al. Outcome of patients with acute promyelocytic leukemia failing to front-line treatment with all-trans retinoic acid and anthracyclinebased chemotherapy (PETHEMA protocols LPA96 and LPA99): benefit of an early intervention. Leukemia 2007 Mar;21(3):446-52. DOI: 10.1038/sj.leu.2404501 